\title{
Distributed Consensus Tracking for Second-Order Nonlinear Multiagent Systems with a Specified Reference State
}

\author{
Guoguang Wen, ${ }^{1}$ Yongguang Yu, ${ }^{1}$ Zhaoxia Peng, $^{2}$ and Ahmed Rahmani ${ }^{3}$ \\ ${ }^{1}$ Department of Mathematics, Beijing Jiaotong University, Beijing 100044, China \\ ${ }^{2}$ School of Transportation Science and Engineering, Beihang University, Beijing 100191, China \\ ${ }^{3}$ LAGIS UMR 8219 CNRS, Ecole Centrale de Lille, Villeneuve d'Ascq 59651, France
}

Correspondence should be addressed to Zhaoxia Peng; zhaoxiapeng1986@gmail.com

Received 17 February 2014; Revised 1 May 2014; Accepted 4 May 2014; Published 28 May 2014

Academic Editor: Jun-Juh Yan

Copyright (C) 2014 Guoguang Wen et al. This is an open access article distributed under the Creative Commons Attribution License, which permits unrestricted use, distribution, and reproduction in any medium, provided the original work is properly cited.

\begin{abstract}
This paper mainly addresses the distributed consensus tracking problem for second-order nonlinear multiagent systems with a specified reference trajectory. The dynamics of each follower consists of two terms: nonlinear inherent dynamics and a simple communication protocol relying only on the position and velocity information of its neighbors. The consensus reference is taken as a virtual leader, whose output is only its position and velocity information that is available to only a subset of a group of followers. To achieve consensus tracking, a class of nonsmooth control protocols is proposed which reply on the relative information among the neighboring agents. Then some corresponding sufficient conditions are derived. It is shown that if the communication graph associated with the virtual leader and followers is connected at each time instant, the consensus can be achieved at least globally exponentially with the proposed protocol. Rigorous proofs are given by using graph theory, matrix theory, and Lyapunov theory. Finally, numerical examples are presented to illustrate the theoretical analysis.
\end{abstract}

\section{Introduction}

Consensus problem, one of the most important and fundamental issues in the cooperative control, has attracted great attention from researchers in recent years because of broad applications in various real-world multiagent systems, such as cooperative control of unmanned (air) vehicles, formation control of robots and aircrafts, and design of sensor networks, to name a few (see the survey papers [1-7] and extensive references therein). The consensus of multiagent systems means to design a network distributed control policy based on the local information obtained by each agent, such that all agents reach an agreement on certain quantities of interest by negotiating with their neighbors. The quantities of interest might represent attitude, position, velocity, temperature, voltage, and so on, depending on different applications.

Numerous interesting results for consensus problem have been obtained in the past decade. In 1986, Reynolds [8] fist proposed a computer animation model to simulate collective behaviors of multiple agents. In [9], Vicsek et al. proposed a discrete-time distributed model to simulate a group of autonomous agents moving in the plane with the same speed but different heading. In [10], Jadbabaie et al. gave a theoretical explanation for the consensus behavior of Vicsek's model based on graph and matrix theories. Ren and Beard [11] extend the work of Jadbabaie et al. [10] to the case of directed graphs and gave some more relaxed conditions. Olfati-Saber and Murray [1] investigated a systematical framework of consensus problems with directed interconnection graphs or time-delay by Lyapunov-based approach.

Note from the literatures which were concerned with consensus problem that the multiagent systems can usually be classified into leaderless and leader-follower systems. In a leaderless consensus problem [12-14], there does not exist a (virtual) leader, while in a leader-following consensus problem [15-19], there exists a (virtual) leader. The (virtual) leader is a special agent whose motion is independent of all the other agents and thus is followed by all the other ones. Therefore, in recent literatures, a (virtual) leader-follower approach has been widely used to the consensus problem [20-25]. Such a consensus problem with a dynamic (virtual) leader is commonly called consensus tracking problem. Most 
of the above works are based on the first-order control protocols that control the velocities of agents rather than their accelerations (which are commonly easier to control and are especially meaningful for analysis of dynamical system in the real world). Hence, it is necessary to study the consensus tracking problem of second-order multiagent systems. In [26], the consensus tracking problem of multiagent systems with double-integrator dynamics was studied. However, [26] requires the availability of the virtual leader's acceleration input to all followers. Compared with previous works of [26]. Reference [20] studied a distributed leader-following consensus problem with single-integrator dynamics and double-integrator dynamics under fixed and switching communication topologies. It was shown that the acceleration measurements of the virtual leader and followers are not required, and the consensus tracking can be achieved in finite time. In [27], the authors studied the consensus in multiagent systems with second-order dynamics and sampled data. Note also from the above literatures that most of common models used to study the consensus tracking of the second-order multiagent systems are double integrator models. However, in reality, mobile agents may be governed by more commonly inherent nonlinear dynamics $[15,28-31]$. References [15, 28] studied the leaderless consensus problems for secondorder multiagent systems with nonlinear dynamics under fixed network topology. Reference [32] investigated secondorder leader-follower consensus of nonlinear multiagent systems via pinning control. Reference [33] investigated leaderfollowing consensus for second-order multiagent systems with nonlinear inherent dynamics. However, in the above literatures each agent's state only depends on their common inherent dynamics. It is difficult to track a time-varying desired reference state for all agents.

Motivated by the above discussions, the main contributions of this paper lie in that we deal with the consensus tracking for second-order nonlinear multiagent systems with a specified reference state. The dynamics of each follower consists of two terms: nonlinear inherent dynamics and a simple communication protocol relying only on the position and velocity information of its neighbors. The consensus reference is taken as a virtual leader, whose output is only its position and velocity information that is available to only a subset of a group of followers. To achieve consensus tracking, a class of nonsmooth control protocols is proposed which reply on the relative information among the neighboring agents. Then some corresponding sufficient conditions are derived. It is shown that if the communication graph associated with the virtual leader and followers is connected at each time instant, the consensus can be achieved at least globally exponentially with the proposed protocol. Rigorous proofs are given by using graph theory, matrix theory, and Lyapunov theory. Compared with the existing results, this paper has the following advantages. Firstly, with contrast of consensus for multiagent systems with double-integrator dynamics [19, 20, 25-27], we investigate the consensus tracking problems for multiagent systems with nonlinear inherent dynamics. Secondly, in contrast to the existing results in $[15,28,32,33]$, where each agent's state only depends on their common inherent dynamics, in this paper all agents can well track a time varying desired reference state. Thirdly, in this paper the consensus can be achieved globally exponentially with the proposed protocol, while in $[15,27-31]$ the consensus can be archived asymptotically. Finally, it is well known that most of real-world systems, for example, biological systems, autonomous vehicles systems, complex systems, and so on, are time-varying systems $[34,35]$. Therefore, in this paper, we consider the consensus tracking problems for time-varying multiagent systems, where the states of all agents are time varying.

The rest of this paper is organized as follows. In Section 2, the relevant notations and preliminaries are presented. In Section 3, the consensus tracking problem to be solved in this paper is described. The main results are presented in Section 4. Numerical examples are shown in Section 5. Finally, some conclusion remakes are given in Section 6.

\section{Preliminaries}

2.1. Notations. The following notations will be used throughout this paper: Let $I_{n}$ denote the $n \times n$ identity matrix, let $0_{m \times n}$ denote the $m \times n$ zero matrix, and let $\mathbf{1}_{n}=[1,1, \ldots, 1]^{T} \epsilon$ $R^{n}$ (1 for short, when there is no confusion). $\lambda_{\min }(A)$ and $\lambda_{\max }(A)$ are the smallest and the largest eigenvalues of the matrix $A$, respectively.

2.2. Graph Theory Notions. Using graph theory, we can model the interaction topology in multiagent systems consisting of $n$ agents. Let $\mathscr{G}=(\mathscr{V}, \mathscr{C}, \mathscr{A})$ be a weighted graph of order $n$ with the finite nonempty set of nodes $\mathscr{V}=\left\{v_{1}, \ldots, v_{n}\right\}$, the set of edges $\mathscr{E} \subseteq \mathscr{V} \times \mathscr{V}$, and a weighted adjacency matrix $\mathscr{A}=\left(a_{i j}\right)_{n \times n}$. Here, each node $v_{i}$ in $\mathscr{V}$ corresponds to an agent $i$, and each edge $\left(v_{i}, v_{j}\right) \in \mathscr{E}$ in a weighted directed graph corresponds to an information link from agent $j$ to agent $i$, which means that agent $i$ can receive information from agent $j$. In contrast, the pairs of nodes in weighted undirected graph are unordered, where an edge $\left(v_{j}, v_{i}\right) \in \mathscr{E}$ denotes that agent $i$ and $j$ can receive information from each other. The weighted adjacency matrix $\mathscr{A}$ of a weighted directed graph is defined such that $a_{i i}=0$ for any $v_{i} \in \mathscr{V}, a_{i j}>0$ if $\left(v_{j}, v_{i}\right) \in \mathscr{E}$, and $a_{i j}=0$ otherwise. The weighted adjacency matrix $\mathscr{A}$ of a weighted undirected graph is defined analogously except that $a_{i j}=a_{j i}$, for all $i \neq j$, since $\left(v_{i}, v_{j}\right) \in \mathscr{E}$ implies $\left(v_{j}, v_{i}\right) \in \mathscr{E}$. We can say $v_{i}$ is a neighbor vertex of $v_{j}$, if $\left(v_{i}, v_{j}\right) \in \mathscr{E}$.

The Laplacian matrix $L=\left(l_{i j}\right)_{n \times n}$ of graph $\mathscr{G}$ is defined by $l_{i j}=-a_{i j}$ for $i \neq j$, and $l_{i i}=\sum_{j=1, j \neq i}^{n} a_{i j}, i, j \in\{1, \ldots, n\}$. For an undirected graph, $L$ is symmetric positive semidefinite. However, $L$ is not necessarily symmetric for a directed graph.

Lemma 1 (see [36]). Assume $\mathscr{G}$ is a weighted undirected graph with Laplacian matrix $L$; then the following two statements are equivalent:

(i) the matrix L has an eigenvalue zero with multiplicity 1 and corresponding eigenvector $\mathbf{1}$, and all other eigenvalues are positive;

(ii) $\mathscr{G}$ is connected. 
2.3. Nonsmooth Analysis Background. Consider the following vector differential equation:

$$
\dot{x}(t)=f(x(t)),
$$

where $x(t)=\left[x_{1}(t), x_{2}(t), \ldots, x_{n}(t)\right]^{T}$ and $f(x(t))$ is not necessarily continuous. A Filippov solution of (1) on $\left[t_{0}, t_{1}\right]$, where $t_{1}$ could be $\infty$, is absolutely continuous function $x(t)$ satisfying the following differential inclusion:

$$
\dot{x}(t) \in \mathscr{K}[f](x(t)),
$$

where $\mathscr{K}[f](x(t)) \triangleq \bigcap_{\delta>0} \bigcap_{\mu(N)=0} \overline{\operatorname{co}} f(t, \mathscr{B}(x, \delta) \backslash N)$, $\bigcap_{\mu(N)=0}$ denotes the intersection over all sets of Lebesgue measure zeroes, $\mathscr{B}(x, \delta)$ denotes the open ball of radius $\delta$ centered at $x$, and $\overline{\mathrm{co}}(\Omega)$ denotes the convex closure of the convex hull of the set $\Omega$.

Definition 2 (see [37]). For a locally Lipschitz continuous function $V$, define the Clarke's generalized gradient of $V$ by $\partial V \triangleq \operatorname{co}\left\{\lim \nabla V(x) \mid x_{i} \rightarrow x, x_{i} \in \Omega_{v} \cup \bar{N}\right\}$, where co denotes the convex hull, $\Omega_{v}$ is the set of Lebesgue measure zero, where $\nabla V$ does not exist, and $\bar{N}$ is an arbitrary set of zero measure. The set-valued Lie derivative of $V$ with respect to (1) is defined as

$$
\mathscr{L}_{\mathscr{K}} V \triangleq \bigcap_{\xi \in \partial V} \xi^{T} \mathscr{K}[f](x(t)) .
$$

In the following, a Lyapunov stability theorem in terms of the set-valued map $\mathscr{L}_{\mathscr{K}} V$ is stated.

Lemma 3 (see [38]). Given (1), let $f(x(t))$ be locally essentially bounded and $0 \in \mathscr{K}[f](0)$ in a region $Q \supset\left\{x \in \mathbb{R}^{m} \mid\|x\|<\right.$ $r\} \times t \mid t_{0} \leq t<\infty$, where $r>0$. Also, let $V: \mathbb{R}^{d} \rightarrow \mathbb{R}$ be a regular function satisfying $V(0, t)=0$ and $0<V_{1}(\|x\|) \leq$ $V \leq V_{2}(\|x\|)$, for $z \neq 0$, in $Q$ for some $V_{1}$ and $V_{2}$ belonging to class $\mathscr{K}$. If there exists a class $\mathscr{K}$ function $w(\cdot)$ in $Q$ such that the set-valued Lie derivative of $V(x, t)$ satisfies

$$
\max \mathscr{L}_{\mathscr{K}} V \leq-w(x)<0, \quad \text { for } x \neq 0,
$$

then the solution $x(t) \equiv 0$ is asymptotically stable.

\section{Problem Description}

Consider a multiagent system consisting of $n$ agents. In what follows, all agents are assumed in one-dimensional space for the simplicity of presentation. However, all results hereafter are still valid for the $m$-dimensional $(m>1)$ by the Kronecker product [39]. Here, the dynamics of each agent in the group is given by

$$
\begin{gathered}
\dot{\xi}_{i}=v_{i}, \\
\dot{v}_{i}=f\left(t, \xi_{i}, v_{i}\right)+u_{i},
\end{gathered}
$$

where $i(i=1, \ldots, n), \xi_{i} \in R^{m}$ and $v_{i} \in R^{m}$ denote the position and velocity vectors, $f\left(t, \xi_{i}, v_{i}\right) \in R^{m}$ denotes the inherent nonlinear dynamics, and $u_{i}(t)$ denotes the control input. When $f\left(t, \xi_{i}, v_{i}\right) \equiv 0$, the multiagent system has double-integrator dynamics.
The consensus problem of the multiagent system (5) is to design control inputs $u_{i}, i=\{1, \ldots, n\}$ such that

$$
\begin{aligned}
& \lim _{t \rightarrow \infty}\left\|\xi_{i}-\xi_{0}\right\|_{2}=0, \\
& \lim _{t \rightarrow \infty}\left\|v_{i}-v_{0}\right\|_{2}=0,
\end{aligned}
$$

for any $i=1,2, \ldots, n$, where $\xi_{0} \in R^{m}$ and $v_{0} \in R^{m}$ are, respectively, the position vector and velocity vector of the virtual leader, which does not have to be an actual agent but is specified by

$$
\begin{gathered}
\dot{\xi}_{0}=v_{0}, \\
\dot{v}_{0}=f\left(t, \xi_{0}, v_{0}\right)+g\left(t, \xi_{0}, v_{0}\right),
\end{gathered}
$$

where $f\left(t, \xi_{0}, v_{0}\right) \in R^{m}$ describes the nonlinear dynamics of the virtual leader, and $g\left(t, \xi_{0}, v_{0}\right) \in R^{m}$ is responsible for controlling trajectory of the virtual leader. We assume that $\left|g\left(t, \xi_{0}, v_{0}\right)\right| \leq C_{0}$, where $C_{0}$ is a positive constant.

Hereafter, the $n$ agents in systems (5) are called followers, and their communication topology is represented by the graph $\mathscr{G}$. Suppose that the virtual leader and all followers share the same nonlinear inherent dynamics, and these nonlinear inherent dynamics satisfy a Lipchitz-type condition given by Assumption 4 as follows, which is satisfied in many well-known systems.

Assumption 4. There exist two nonnegative constants $l_{1}$ and $l_{2}$ such that

$$
\begin{array}{r}
\|f(t, \xi, v)-f(t, \zeta, \gamma)\|_{2} \leq l_{1}\|\xi-\zeta\|_{2}+l_{2}\|v-\gamma\|_{2}, \\
\forall \xi, v, \zeta, \gamma \in R^{m}, \quad \forall t \geq 0 .
\end{array}
$$

Remark 5. Note from Assumption 4 that the Lipchitz constants $l_{1}$ or $l_{2}$ may be chosen by zero. In order to satisfy the requirements of designing consensus protocols, in this paper we assume that $r_{1}=\left\lfloor l_{1}\right\rfloor+1$ and $r_{2}=\left\lfloor l_{2}\right\rfloor+1$, where $\lfloor\cdot\rfloor$ denotes floor function (i.e., $\lfloor x\rfloor=\max \{m \in \mathbb{Z} \mid m \leq x\}$, where $\mathbb{Z}$ is the set of integers).

Remark 6. In recent reference [40], the robust cooperative tracking for multiple nonidentical second-order nonlinear systems is investigated. The nonlinear inherent dynamics $f\left(t, \xi_{i}, v_{i}\right)$ in this paper satisfies the Lipchitz condition (8), while the nonlinear function $f\left(t, \xi_{i}, v_{i}\right)$ in [40] is required to be continuously differentiable (the derivative $\dot{f}\left(t, \xi_{i}, v_{i}\right)$ exists in its domain and is itself a continuous function).

\section{Consensus Tracking Protocols}

To satisfy (6), the following control input (9) is proposed for each follower:

$$
\begin{aligned}
u_{i}(t)= & -\alpha \sum_{j=0}^{n} a_{i j}\left[r_{1}\left(\xi_{i}-\xi_{j}\right)+r_{2}\left(v_{i}-v_{j}\right)\right] \\
& -\beta \operatorname{sgn}\left\{\sum_{j=0}^{n} a_{i j}\left[r_{1}\left(\xi_{i}-\xi_{j}\right)+r_{2}\left(v_{i}-v_{j}\right)\right]\right\},
\end{aligned}
$$


where $\alpha$ is a nonnegative constant, $\beta$ is a positive constant, $a_{i j}, i, j=1, \ldots, n$, is the $(i, j)$ th entry of the adjacency matrix $\mathscr{A}$ associated with $G$, and $\operatorname{sgn}(\cdot)$ is the signum function. In addition, $a_{i 0}>0(i=1, \ldots, n)$ if the virtual leader's position is available to follower $i$, and $a_{i 0}=0$ otherwise.

Substituting (9) to (5) gives

$$
\begin{gathered}
\dot{\xi}_{i}=v_{i}, \\
\dot{v}_{i}=f\left(t, \xi_{i}, v_{i}\right)-\alpha \sum_{j=0}^{n} a_{i j}\left[r_{1}\left(\xi_{i}-\xi_{j}\right)+r_{2}\left(v_{i}-v_{j}\right)\right] \\
-\beta \operatorname{sgn}\left\{\sum_{j=0}^{n} a_{i j}\left[r_{1}\left(\xi_{i}-\xi_{j}\right)+r_{2}\left(v_{i}-v_{j}\right)\right]\right\} .
\end{gathered}
$$

Let $M=L+\operatorname{diag}\left(a_{10}, \ldots, a_{n 0}\right)$, where $L$ is the Laplacian matrix of $\mathscr{G}$.

Lemma 7. Suppose that the communication graph $\mathscr{G}$ is connected and at least one follower can receive information from the virtual leader. Let $H=\left[\begin{array}{cc}\left(\alpha r_{2} / 2 r_{1}\right) M^{2} & \left(1 / 2 r_{2}\right) M \\ \left(1 / 2 r_{2}\right) M & \left(1 / 2 r_{2}\right) M\end{array}\right]$ and $Q=\left[\begin{array}{cc}\alpha M^{2} & (1 / 2) \alpha M^{2} \\ (1 / 2) \alpha M^{2} & \alpha M^{2}-\left(r_{1} / r_{2}^{2}\right) M\end{array}\right]$. If $\alpha>\left(8 r_{2}^{2}+2 r_{1}+\right.$ $2 \sqrt{\widehat{\Omega}}) \lambda_{\max }(M) / 3 r_{2}^{2} \lambda_{\min }^{2}(M)$, then the matrices $H$ and $Q$ are symmetric positive definite, and $\lambda_{\min }(Q)>2 \lambda_{\max }(M)$, where $\widehat{\Omega}=4 r_{2}^{4}+2 r_{1} r_{2}^{2}+r_{1}^{2}$.

Proof. Since the communication graph $\mathscr{G}$ is connected and the virtual leader is a neighbor of at least one follower, it follows that the matrix $M$ is symmetric positive definite. Therefore, the matrix $M$ can be diagonalized as $M=T^{-1} \Delta T$, where $\Delta=\left\{\lambda_{1}, \lambda_{2}, \ldots, \lambda_{n}\right\}, \lambda_{i}>0$ with $\lambda_{i}$ being the $i$ th eigenvalue of $M$. Then we can obtain a matrix $\Pi$ such that

$$
\Pi=\left[\begin{array}{cc}
\frac{\alpha r_{2}}{2 r_{1}} \Delta^{2} & \frac{1}{2 r_{2}} \Delta \\
\frac{1}{2 r_{2}} \Delta & \frac{1}{2 r_{2}} \Delta
\end{array}\right]=\left[\begin{array}{cc}
T & \mathbf{0}_{n \times n} \\
\mathbf{0}_{n \times n} & T
\end{array}\right] H\left[\begin{array}{cc}
T^{-1} & \mathbf{0}_{n \times n} \\
\mathbf{0}_{n \times n} & T^{-1}
\end{array}\right],
$$

where $\mathbf{0}_{n \times n}$ is the $n \times n$ zero matrix. It is easy to see that $\Pi$ is symmetric and has the same eigenvalues as $H$.

Let $\delta$ be an eigenvalue of $\Pi$. Because $\Delta$ is a diagonal matrix, it follows from (11) that

$$
\delta^{2}-\frac{\alpha r_{2}^{2} \lambda_{i}^{2}+r_{1} \lambda_{i}}{2 r_{1} r_{2}} \delta+\frac{\alpha r_{2}^{2} \lambda_{i}^{3}-r_{1} \lambda_{i}^{2}}{4 r_{1} r_{2}^{2}}=0 .
$$

As the matrix $\Pi$ is symmetric, it follows that the roots of (12) are all real. Therefore, they are positive if and only if the following conditions are satisfied:

$$
\frac{\alpha r_{2}^{2} \lambda_{i}^{2}+r_{1} \lambda_{i}}{2 r_{1} r_{2}}>0, \quad \frac{\alpha r_{2}^{2} \lambda_{i}^{3}-r_{1} \lambda_{i}^{2}}{4 r_{1} r_{2}^{2}}>0
$$

And, $H$ is positive definite if

$$
\alpha>\frac{r_{1}}{r_{2}^{2} \lambda_{\min }(M)} .
$$

Next, we consider the matrix $Q$. Similarly with the analysis of the matrix $H$, there exists a matrix $J$ such that

$$
J=\left[\begin{array}{cc}
\alpha \Delta^{2} & \frac{1}{2} \alpha \Delta^{2} \\
\frac{1}{2} \alpha \Delta^{2} & \alpha \Delta^{2}-\frac{r_{1}}{r_{2}^{2}} \Delta
\end{array}\right]=\left[\begin{array}{cc}
T & \mathbf{0}_{n \times n} \\
\mathbf{0}_{n \times n} & T
\end{array}\right] Q\left[\begin{array}{cc}
T^{-1} & \mathbf{0}_{n \times n} \\
\mathbf{0}_{n \times n} & T^{-1}
\end{array}\right] .
$$

$J$ is symmetric and has the same eigenvalues as $Q$.

Let $\varepsilon$ be an eigenvalue of $J$. Because $\Delta$ is a diagonal matrix, it follows from (15) that $\varepsilon$ satisfies

$$
\varepsilon^{2}-\left[2 \alpha \lambda_{i}^{2}-\frac{r_{1}}{r_{2}^{2}} \lambda_{i}\right] \varepsilon+\alpha \lambda_{i}^{2}\left(\alpha \lambda_{i}^{2}-\frac{r_{1}}{r_{2}^{2}} \lambda_{i}\right)-\frac{1}{4} \alpha^{2} \lambda_{i}^{4}=0 .
$$

Therefore, $\lambda_{\min }(Q)>2 \lambda_{\max }(M)$ if and only if the following inequations are satisfied:

$$
\begin{gathered}
\frac{2 \alpha \lambda_{i}^{2}-\left(r_{1} / r_{2}^{2}\right) \lambda_{i}}{2}>2 \lambda_{\max }(M) \\
\left(2 \lambda_{\max }(M)\right)^{2}-\left[2 \alpha \lambda_{i}^{2}-\frac{r_{1}}{r_{2}^{2}} \lambda_{i}\right]\left(2 \lambda_{\max }(M)\right) \\
+\alpha \lambda_{i}^{2}\left(\alpha \lambda_{i}^{2}-\frac{r_{1}}{r_{2}^{2}} \lambda_{i}\right)-\frac{1}{4} \alpha^{2} \lambda_{i}^{4}>0
\end{gathered}
$$

From (17), it follows that

$$
\alpha>\frac{\left(4 r_{2}^{2}+r_{1}\right) \lambda_{\max }(M)}{2 r_{2}^{2} \lambda_{\min }^{2}(M)} \geq \frac{4 r_{2}^{2} \lambda_{\max }(M)+r_{1} \lambda_{i}}{2 r_{2}^{2} \lambda_{i}^{2}} .
$$

From (18), it follows that

$$
\alpha>\frac{8 r_{2}^{2} \lambda_{\max }(M)+2 r_{1} \lambda_{i}+2 \sqrt{\Omega}}{3 r_{2}^{2} \lambda_{i}^{2}}
$$

or

$$
\alpha<\frac{8 r_{2}^{2} \lambda_{\max }(M)+2 r_{1} \lambda_{i}-2 \sqrt{\Omega}}{3 r_{2}^{2} \lambda_{i}^{2}},
$$

where $\Omega=4 r_{2}^{4} \lambda_{\max }^{2}(M)+2 r_{1} r_{2}^{2} \lambda_{i} \lambda_{\max }(M)+r_{1}^{2} \lambda_{i}^{2}$. if

Therefore, $Q$ is positive definite and $\lambda_{\min }(Q)>2 \lambda_{\max }(M)$

$$
\alpha>\frac{\left(8 r_{2}^{2}+2 r_{1}+2 \sqrt{\widehat{\Omega}}\right) \lambda_{\max }(M)}{3 r_{2}^{2} \lambda_{\min }^{2}(M)},
$$

where $\widehat{\Omega}=4 r_{2}^{4}+2 r_{1} r_{2}^{2}+r_{1}^{2}$.

Combining the results (14) and (22), the proof is completed. 
Theorem 8. Suppose that the communication graph $\mathscr{G}$ is connected and the virtual leader is a neighbor of at least one follower. If $\alpha>\left(8 r_{2}^{2}+2 r_{1}+2 \sqrt{\widehat{\Omega}}\right) \lambda_{\max }(M) / 3 r_{2}^{2} \lambda_{\text {min }}^{2}(M)$ and $\beta>C_{0}$, then second-order consensus tracking in system (10) is achieved at least globally exponentially, where $\widehat{\Omega}=4 r_{2}^{4}+2 r_{1} r_{2}^{2}+$ $r_{1}^{2}$.

Proof. Let $\tilde{\xi}_{i}=\xi_{i}-\xi_{0}, \widetilde{v}_{i}=v_{i}-v_{0}, i \in\{1, \ldots, n\}$. From (7) and (10),

$$
\begin{gathered}
\dot{\widetilde{\xi}}_{i}=\widetilde{v}_{i}, \\
\dot{\tilde{v}}_{i}=-\alpha \sum_{j=0}^{n} a_{i j}\left[r_{1}\left(\widetilde{\xi}_{i}-\widetilde{\xi}_{j}\right)+r_{2}\left(\widetilde{v}_{i}-\widetilde{v}_{j}\right)\right] \\
-\beta \operatorname{sgn}\left\{\sum_{j=0}^{n} a_{i j}\left[r_{1}\left(\widetilde{\xi}_{i}-\widetilde{\xi}_{j}\right)+r_{2}\left(\widetilde{v}_{i}-\widetilde{v}_{j}\right)\right]\right\} \\
+f\left(t, \xi_{i}, v_{i}\right)-f\left(t, \xi_{0}, v_{0}\right)+g\left(t, \xi_{0}, v_{0}\right) .
\end{gathered}
$$

Let $\tilde{\xi}=\left[\tilde{\xi}_{1}^{T}, \widetilde{\xi}_{2}^{T}, \ldots, \widetilde{\xi}_{n}^{T}\right]^{T}, \widetilde{v}=\left[\widetilde{v}_{1}^{T}, \widetilde{v}_{2}^{T}, \ldots, \widetilde{v}_{n}^{T}\right]^{T}$ and $F(t, \widetilde{\xi}, \widetilde{v})=$ $\left[\left(f\left(t, \xi_{1}, v_{1}\right)-f\left(t, \xi_{0}, v_{0}\right)\right)^{T}, \ldots,\left(f\left(t, \xi_{n}, v_{n}\right)-f\left(t, \xi_{0}, v_{0}\right)\right)^{T}\right]^{T}$. Rewrite (23) in the matrix form as

$$
\begin{gathered}
\dot{\vec{\xi}}=\widetilde{v}, \\
\dot{\tilde{v}}=F(t, \widetilde{\xi}, \widetilde{v})-\alpha M\left(r_{1} \widetilde{\xi}+r_{2} \widetilde{v}\right)-\beta \operatorname{sgn}\left(M\left(r_{1} \widetilde{\xi}+r_{2} \widetilde{v}\right)\right) \\
+1 g\left(t, \xi_{0}, v_{0}\right) .
\end{gathered}
$$

Consider the Lyapunov function candidate

$$
V(t)=\left[\begin{array}{ll}
r_{1} \widetilde{\xi}^{T} & r_{2} \widetilde{v}^{T}
\end{array}\right] H\left[\begin{array}{l}
r_{1} \tilde{\xi} \\
r_{2} \widetilde{v}
\end{array}\right]
$$

According to Definition 2, in the following let us compute the set-valued lie derivative of $V$ as

$$
\begin{aligned}
\mathscr{L}_{\mathscr{K}} V(\widetilde{\xi}, \widetilde{v}) & =\bigcap_{x \in \partial V(\tilde{\xi}, \widetilde{v})} x^{T} \mathscr{K}[f](\widetilde{\xi}, \widetilde{v}) \\
& =\bigcap_{x \in \partial V(\widetilde{\xi}, \widetilde{v})} x^{T} \mathscr{K}\left[r_{2}\left\{F(t, \tilde{\xi}, \widetilde{v})-\alpha M\left(r_{1} \tilde{\xi}+r_{2} \widetilde{v}\right)-\beta \operatorname{sign}\left(M\left(r_{1} \tilde{\xi}+r_{2} \widetilde{v}\right)\right)+1 g\left(t, \xi_{0}, v_{0}\right)\right\}\right],
\end{aligned}
$$

where $\partial V(\widetilde{\xi}, \widetilde{v})$ is the generalized gradient of $V$. Because $V$ is continuously differentiable, $\partial V(\widetilde{\xi}, \widetilde{v})=H\left[\begin{array}{l}r_{1} \tilde{\xi} \\ r_{2} \widetilde{v}\end{array}\right]$. Hence, we have

$$
\begin{aligned}
& \mathscr{L}_{\mathscr{K}} V(\widetilde{\xi}, \widetilde{v})=\left[\begin{array}{l}
r_{1} \tilde{\xi} \\
r_{2} \widetilde{v}
\end{array}\right]^{T} H^{T} \mathscr{K}\left[r_{2}\left\{F(t, \widetilde{\xi}, \widetilde{v})-\alpha M\left(r_{1} \tilde{\xi}+r_{2} \widetilde{v}\right)-\beta \operatorname{sgn}\left(M\left(r_{1} \tilde{\xi}+r_{2} \tilde{v}\right)\right)+1 g\left(t, \xi_{0}, v_{0}\right)\right\}\right] \\
& =\mathscr{K}\left[-\left[\begin{array}{ll}
r_{1} \widetilde{\xi}^{T} & r_{2} \widetilde{v}^{T}
\end{array}\right] Q\left[\begin{array}{l}
r_{1} \tilde{\xi} \\
r_{2} \widetilde{v}
\end{array}\right]-\beta\left\|\left(r_{1} \widetilde{\xi}^{T}+r_{2} \widetilde{v}^{T}\right) M\right\|_{1}+\left(\widetilde{\xi}^{T} r_{1}+\widetilde{v}^{T} r_{2}\right) M F(t, \tilde{\xi}, \widetilde{v})\right. \\
& \left.+\left(r_{1} \tilde{\xi}^{T}+r_{2} \widetilde{v}^{T}\right) \operatorname{M1g}\left(t, \xi_{0}, v_{0}\right)\right] \\
& =\left\{-\left[\begin{array}{ll}
r_{1} \widetilde{\xi}^{T} & r_{2} \widetilde{v}^{T}
\end{array}\right] Q\left[\begin{array}{l}
r_{1} \tilde{\xi} \\
r_{2} \widetilde{v}
\end{array}\right]-\beta\left\|\left(r_{1} \widetilde{\xi}^{T}+r_{2} \widetilde{v}^{T}\right) M\right\|_{1}+\left(\widetilde{\xi}^{T} r_{1}+\widetilde{v}^{T} r_{2}\right) M F(t, \tilde{\xi}, \widetilde{v})\right. \\
& \left.+\left(r_{1} \tilde{\xi}^{T}+r_{2} \widetilde{v}^{T}\right) M \mathbf{1} g\left(t, \xi_{0}, v_{0}\right)\right\}
\end{aligned}
$$

where the fact that $x^{T} \mathscr{K}[\operatorname{sign}(x)]=\mathscr{K}\left[x^{T} \operatorname{sign}(x)\right]=$ $x^{T} \operatorname{sign}(x)=\|x\|_{1}$ has been used as the fact $x^{T} \mathscr{K}[\operatorname{sign}(x)]$ is continuous. It then follows that the set-valued Lie derivative $\mathscr{L}_{\mathscr{K}} V$ is a singleton, whose only element is actually $\dot{V}$. Since 
$\|F(t, \tilde{\xi}, \widetilde{v})\|_{2} \leq\left\|r_{1} \tilde{\xi}\right\|_{1}+\left\|r_{2} \widetilde{v}\right\|_{2},\left|g\left(t, \xi_{0}, v_{0}\right)\right|<C_{0}$, and $\left\|\left(r_{1} \tilde{\xi}^{T}+r_{2} \widetilde{v}^{T}\right) M\right\|_{1}>\left\|\left(r_{1} \widetilde{\xi}^{T}+r_{2} \widetilde{v}^{T}\right) M\right\|_{2}$, it follows that

$$
\begin{aligned}
\max \mathscr{L}_{\mathscr{K}} V= & \dot{V} \leq-\lambda_{\min }(Q)\left(\left\|r_{1} \tilde{\xi}\right\|_{2}^{2}+\left\|r_{2} \widetilde{v}\right\|_{2}^{2}\right) \\
& +\lambda_{\max }(M)\left(\left\|r_{1} \tilde{\xi}\right\|_{2}+\left\|r_{2} \widetilde{v}\right\|_{2}\right)^{2} \\
& -\beta\left\|\left(r_{1} \widetilde{\xi}^{T}+r_{2} \widetilde{v}^{T}\right) M\right\|_{2} \\
& +C_{0}\left\|\left(r_{1} \tilde{\xi}^{T}+r_{2} \widetilde{v}^{T}\right) M\right\|_{2} \\
\leq & -\left[\lambda_{\min }(Q)-2 \lambda_{\max }(M)\right]\left(\left\|r_{1} \tilde{\xi}\right\|_{2}^{2}+\left\|r_{2} \widetilde{v}\right\|_{2}^{2}\right) \\
& -\left(\beta-C_{0}\right)\left\|\left(r_{1} \tilde{\xi}^{T}+r_{2} \widetilde{v}^{T}\right)\right\|_{2} .
\end{aligned}
$$

Note $\alpha>\left(8 r_{2}^{2}+2 r_{1}+2 \sqrt{\widehat{\Omega}}\right) \lambda_{\max }(M) / 3 r_{2}^{2} \lambda_{\min }^{2}(M)$ and $\beta>C_{0}$; it follows from Lemma 7 that $\max \mathscr{L}_{\mathscr{K}} V$ is negative definite. Therefore, $\tilde{\xi}_{i}(t) \rightarrow \mathbf{0}_{n}$ and $\tilde{v}_{i}(t) \rightarrow \mathbf{0}_{n}$ as $t \rightarrow \infty$, where $\mathbf{0}_{n}$ is $n \times 1$ zero vector. Equivalently, it follows that $\xi_{i}(t) \rightarrow \xi_{0}(t)$ and $v_{i}(t) \rightarrow v_{0}(t)$ as $t \rightarrow \infty$.

Next, we prove that the consensus can be achieved at least globally exponentially. Note that

$$
V=\left[\begin{array}{ll}
r_{1} \tilde{\xi}^{T} & r_{2} \widetilde{v}^{T}
\end{array}\right] H\left[\begin{array}{l}
r_{1} \tilde{\xi} \\
r_{2} \widetilde{v}
\end{array}\right] \leq \lambda_{\max }(H)\left(\left\|r_{1} \tilde{\xi}\right\|_{2}^{2}+\left\|r_{2} \widetilde{v}\right\|_{2}^{2}\right) .
$$

From $(28), \dot{V}(t)$ satisfies that

$$
\begin{aligned}
\max \mathscr{L}_{\mathscr{K}} V(t) & =\dot{V}(t) \\
& \leq-\left(\lambda_{\min }(Q)-2 \lambda_{\max }(M)\right)\left(\left\|r_{1} \tilde{\xi}\right\|_{2}^{2}+\left\|r_{2} \widetilde{v}\right\|_{2}^{2}\right) \\
& \leq-\frac{\left(\lambda_{\min }(Q)-2 \lambda_{\max }(M)\right)}{\lambda_{\max }(H)} V(t) .
\end{aligned}
$$

Therefore, $V(t) \leq V(0) e^{-\left(\left(\lambda_{\min }(Q)-2 \lambda_{\max }(M)\right) / \lambda_{\max }(H)\right) t}$. The proof is completed.

Remark 9. In this paper, we assume that there exists a virtual leader, which does not have to be an actual agent and can be a specified reference state. In fact, the conclusion obtained in this paper can also be extended to the leader-less case where there exists no virtual leader. Because the proof is similar to that of Theorem 8 in this paper, it is therefore omitted here.

\section{Numerical Results}

In order to demonstrate the effectiveness of the theoretic results, some simulations are given in this section.

\subsection{Leader-Follower Case}

5.1.1. Dynamics of Agents. Consider a second-order multiagent system consisting of one virtual leader indexed by 0 and

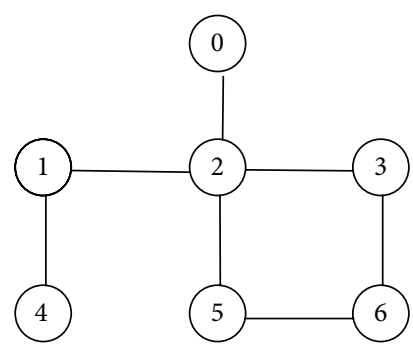

(a)

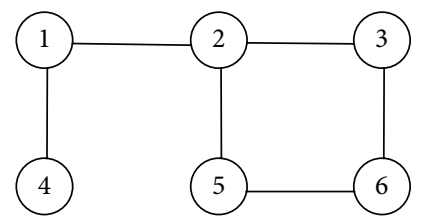

(b)
FIgURE 1: The communication topologies for (a) a group of six followers and one virtual, and (b) a group of six agents without virtual leader.

six followers indexed by 1 to 6 , respectively. The communication topology is given in Figure 1(a). The nonlinear inherent dynamics of each follower is given as follows:

$$
f\left(t, \xi_{i}, v_{i}\right)=\left[\begin{array}{l}
\sin \left(\xi_{i x}\right) \cos (t)+\cos \left(v_{i x}\right) \sin (t) \\
\cos \left(\xi_{i y}\right) \sin (t)+\sin \left(v_{i y}\right) \cos (t)
\end{array}\right] \in R^{2} .
$$

It is easy to verify that $f\left(t, \xi_{i}, v_{i}\right)$ satisfies Assumption 4 . Here, the Lipschitz constants are chosen as $r_{1}=3$ and $r_{2}=3$. The trajectory of virtual leader is chosen as $\xi_{0}(t)=[t, \sin (t)]^{T} \epsilon$ $R^{2}$. It follows that the dynamics of virtual leader is given as

$$
f\left(t, \xi_{0}, v_{0}\right)+g\left(t, \xi_{0}, v_{0}\right)=\left[\begin{array}{c}
0 \\
-\sin (t)
\end{array}\right] \in R^{2} .
$$

Furthermore,

$$
\begin{aligned}
g & \left(t, \xi_{0}, v_{0}\right) \\
\quad & =\left[\begin{array}{c}
-\sin \left(\xi_{i x}\right) \cos (t)-\cos \left(v_{i x}\right) \sin (t) \\
-\cos \left(\xi_{i y}\right) \sin (t)-\sin \left(v_{i y}\right) \cos (t)-\sin (t)
\end{array}\right] .
\end{aligned}
$$

From (33), let us take $C_{0}=3$.

5.1.2. Determinations of $\alpha$ and $\beta$. In this simulation, we suppose that $a_{i j}=1$ if agent $i$ can receive information from agent $j, a_{i j}=0$ otherwise, $i \in\{1, \ldots, n\}$ and $j \in\{0,1, \ldots, n\}$. Therefore, the matrix $M$ can be derived from the topology given in Figure 1(a):

$$
M=\left[\begin{array}{cccccc}
2 & -1 & 0 & -1 & 0 & 0 \\
-1 & 4 & -1 & 0 & -1 & 0 \\
0 & -1 & 2 & 0 & 0 & -1 \\
-1 & 0 & 0 & 1 & 0 & 0 \\
0 & -1 & 0 & 0 & 2 & -1 \\
0 & 0 & -1 & 0 & -1 & 2
\end{array}\right] .
$$

It is easy to obtain that $\lambda_{\text {min }}(M)=0.1284$. By Theorem 8, when $\alpha \leq\left(\left(8 r_{2}^{2}+2 r_{1}\right)+2 \sqrt{\left(r_{1}+r_{2}^{2}\right)^{2}+3 r_{2}^{4}}\right) / 3 r_{2}^{2} \lambda_{\min }(M)=$ 31.4657, and $\beta>C_{0}=3$, the consensus tracking can be achieved. Here we choose $\alpha=32, \beta=4$. 


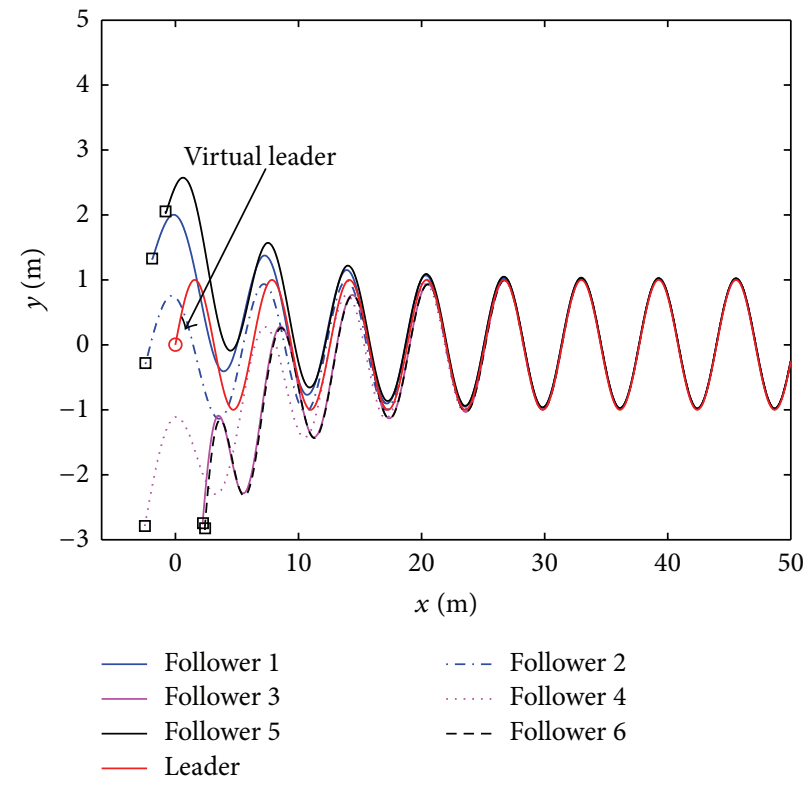

FIgURE 2: Position states of the followers and virtual leader under the communication topology of Figure 1(a).

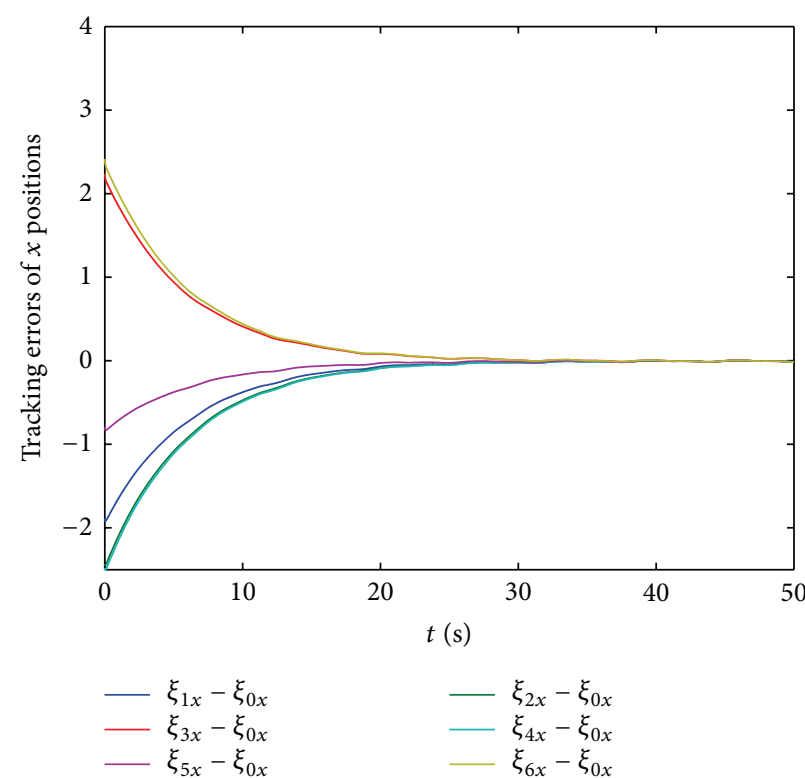

(a)

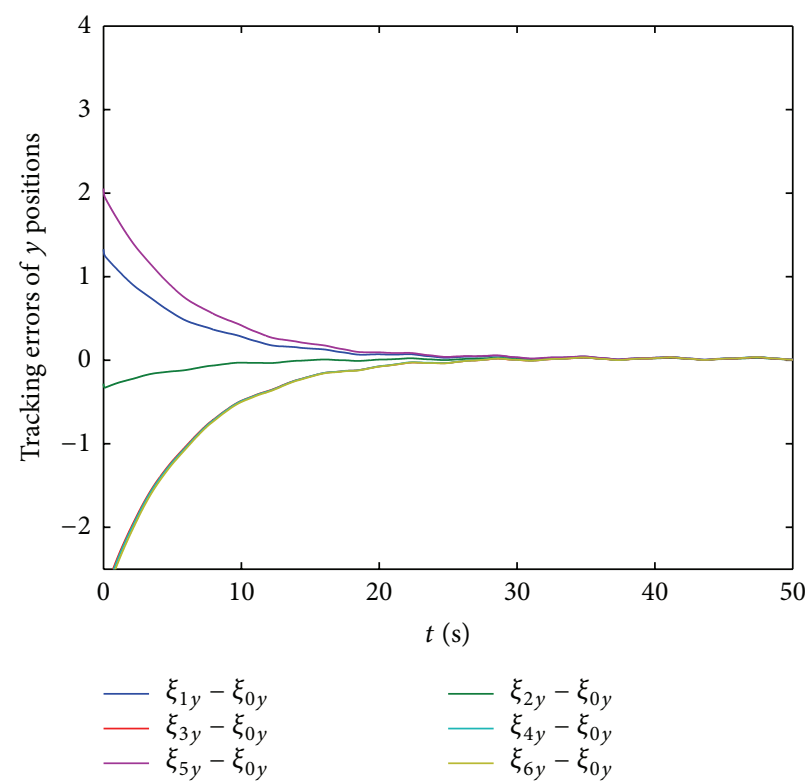

(b)

Figure 3: (a) Position tracking errors along $x$-axis; (b) position tracking errors along $y$-axis.

5.1.3. Simulation Results. Figure 2 shows the position states of the virtual leader and followers. Figures 3(a) and 3(b) show the position tracking errors along $x$-axis and $y$-axis. Figures 4(a) and 4(b) show the velocity tracking errors along $x$-axis and $y$-axis, respectively. Here, the initial position and velocity states of followers are randomly chosen from the cubes $[-3,3] \times[-3,3]$ and $[-2,2] \times[-2,2]$, respectively, and the initial position and velocity states of the virtual leader are $\xi_{0}(0)=[0,0]^{T}$ and $v_{0}(0)=[1,1]^{T}$. It can be seen that all followers ultimately track the virtual leader. Simulation results verify the theoretical analysis very well.
5.2. Leaderless Case. In this part, let us consider a group of six agents without virtual leader. The communication topology is given in Figure 1(b). In order to compare with leader-follower case, the nonlinear inherent dynamics of each agent is chosen as (31), which is the same as that in leader-follower case. $\alpha$ and $\beta$ are also chosen as 32 and 4, respectively; the initial position and velocity states of six agents are still randomly chosen from the cubes $[-3,3] \times[-3,3]$ and $[-2,2] \times[-2,2]$, respectively,

Figure 5 shows the position states of six agents under communication topology in Figure 1(b) . Figures $6(\mathrm{a})$ and $6(\mathrm{~b})$ show the position tracking errors 


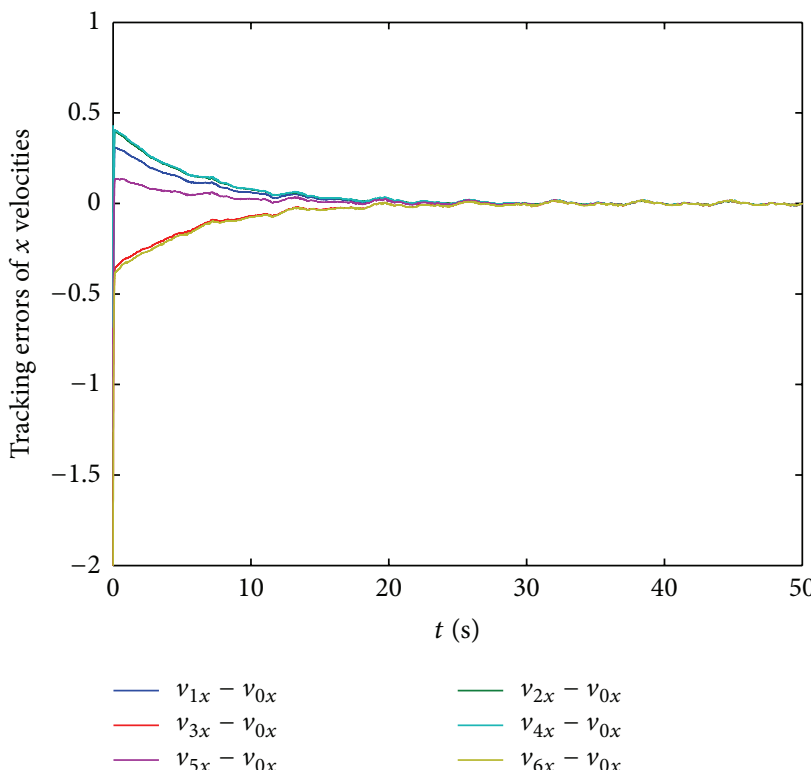

(a)

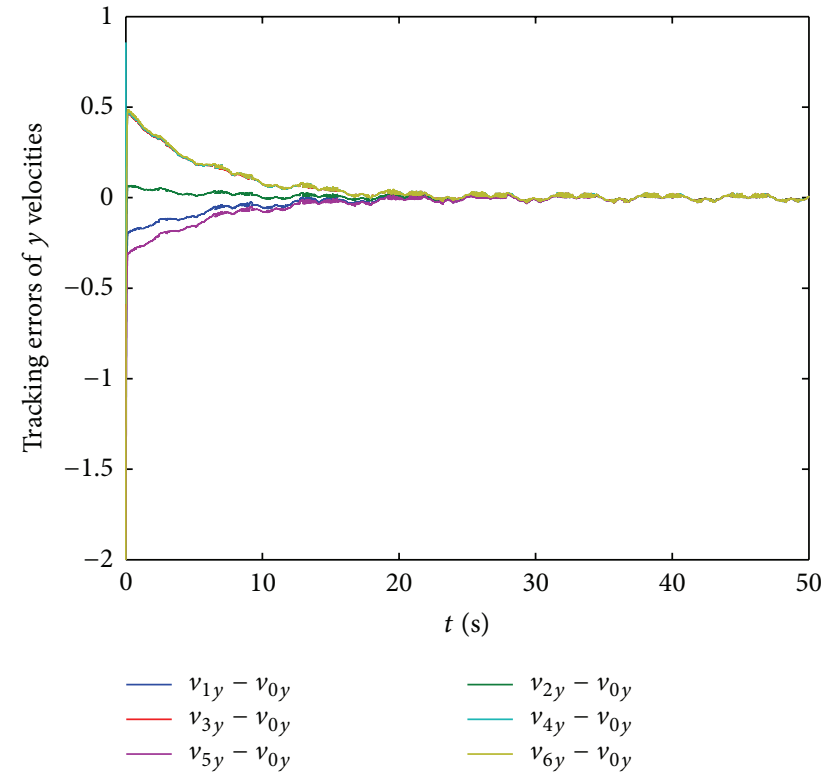

(b)

FIGURE 4: (a) Velocity tracking errors along $x$-axis; (b) velocity tracking errors along $y$-axis.

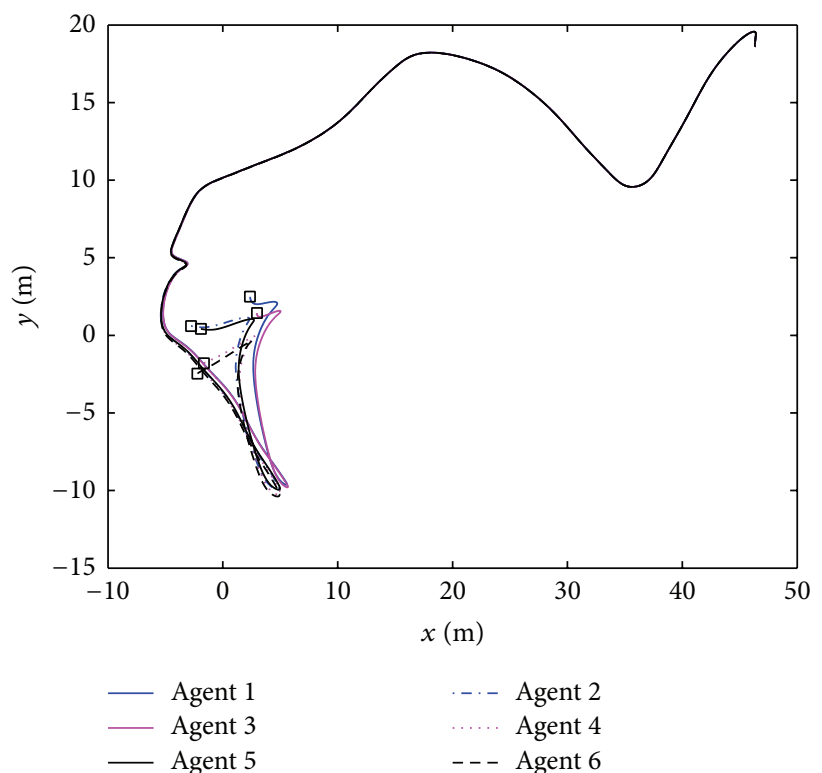

Figure 5: Position states of six agents.

along $x$-axis and $y$-axis. Figures 7 (a) and $7($ b) show the velocity tracking errors along $x$-axis and $y$-axis, respectively. It can be seen from Figures 6 and 7 that the tracking errors ultimately converge to zero. We see from Figure 5 that consensus tracking is achieved.

Remark 10. For the leaderless consensus case, the final states of each agent are determined by the communication topology, the control gains, and the initial value of each agent. The final states of each agent cannot be specified. However, for the leader-follower case, there exists a virtual leader that determines the final state, and the control objective is to guarantee that the final states of all agents reach the state of the virtual leader. From the above simulations, the conclusion obtained in this paper can be extended to the leaderless case.

\section{Conclusion}

The consensus tracking problem for second-order multiagent systems with nonlinear inherent dynamics and a time varying reference state has been studied in this paper. A class of nonsmooth control protocols has been proposed, and the 


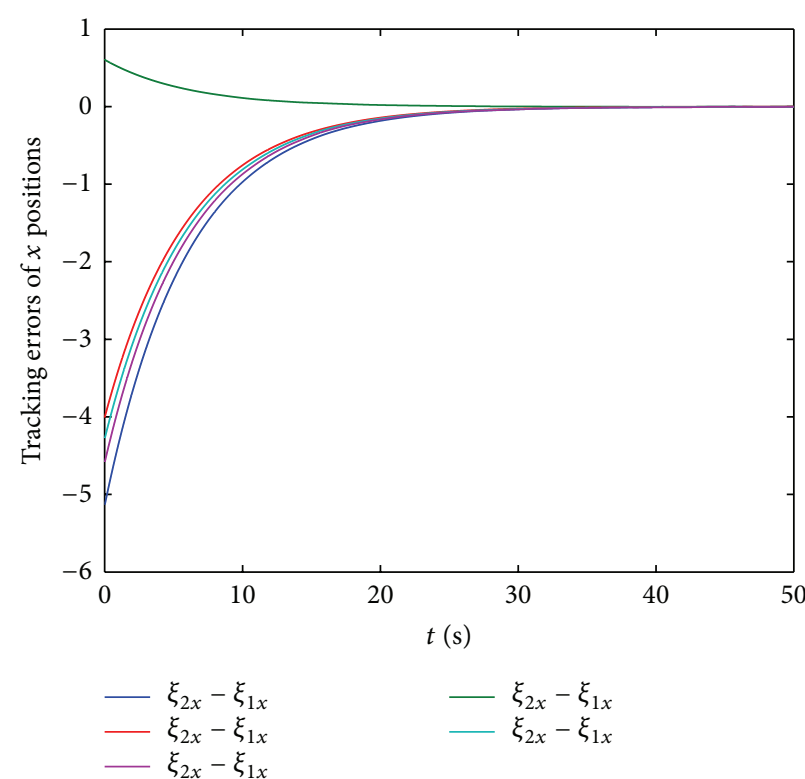

(a)

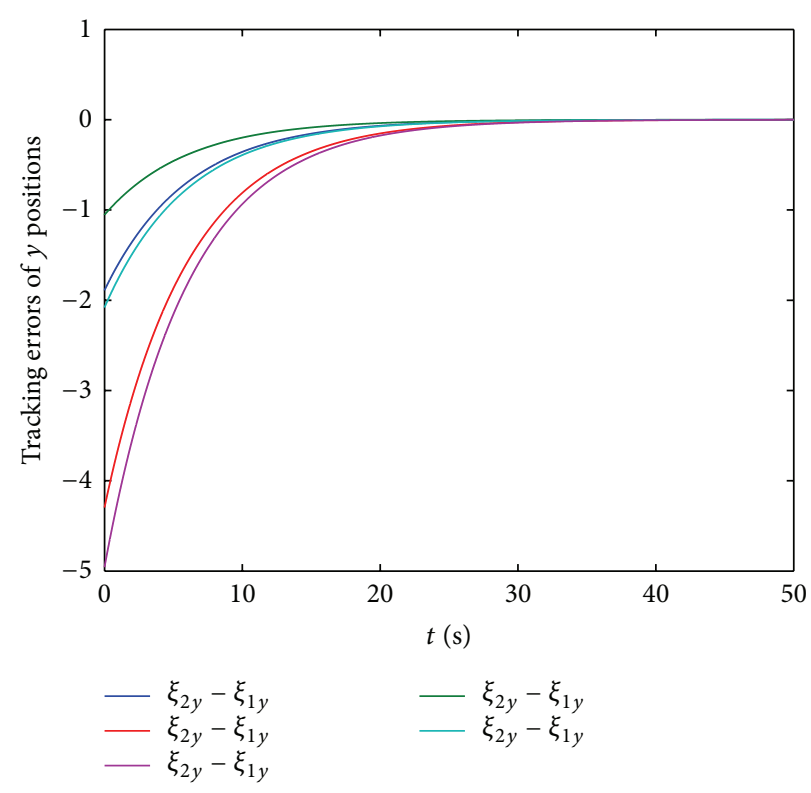

(b)

Figure 6: (a) Position tracking errors along $x$-axis; (b) position tracking errors along $y$-axis.

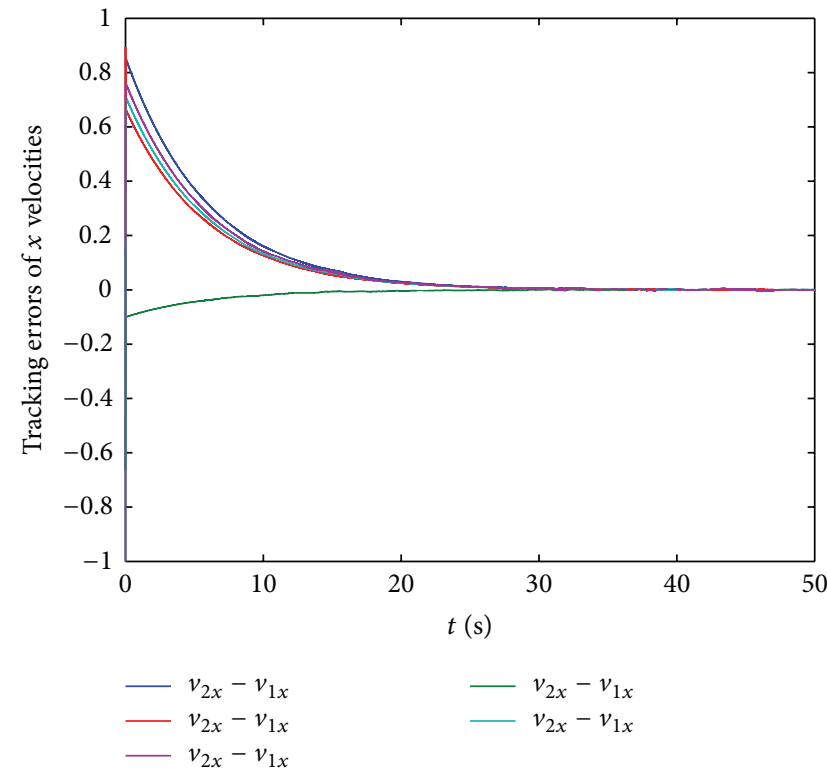

(a)

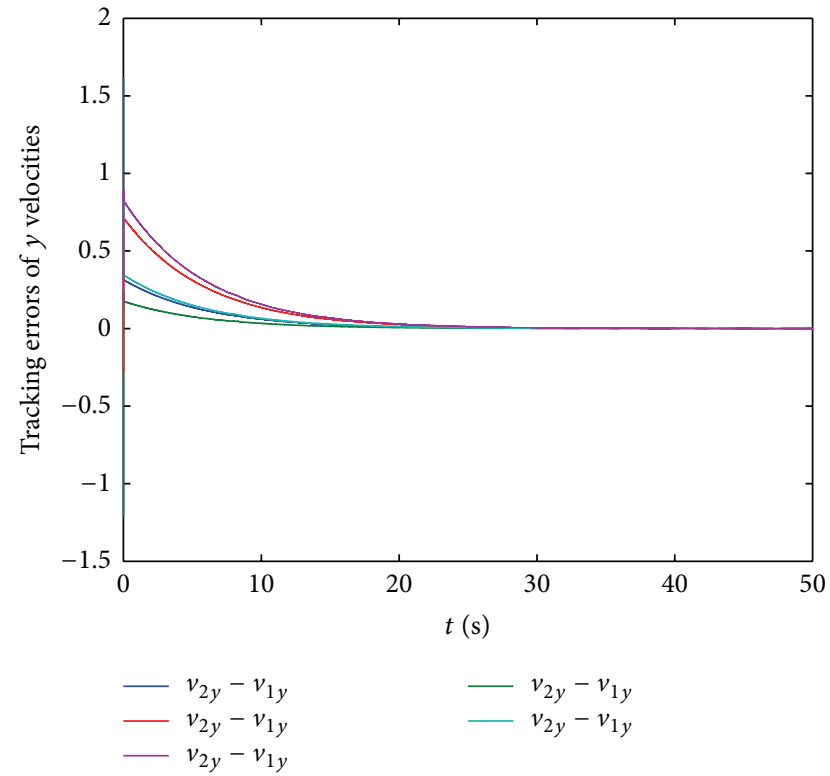

(b)

Figure 7: (a) Velocity tracking errors along $x$-axis; (b) velocity tracking errors along $y$-axis.

corresponding sufficient conditions have been obtained. It is found that if the communication graph associated with the virtual leader and followers is connected, the consensus can be achieved globally exponentially with the proposed protocol.

There are still a number of related interesting problems that deserve further infestation, for example, the consensus tracking problem for high-order multiagent systems with nonlinear inherent dynamics, the consensus tracking problem under directed communication topology and with time delays, and so on, some of which will be studied in the near future.

\section{Conflict of Interests}

The authors declare that there is no conflict of interests regarding the publication of this paper.

\section{Acknowledgments}

This work was supported by the Fundamental Research Funds for the Central Universities (no. YWF-14-RSC-032), the National Nature Science Foundation of China under Grant no. 11371049, and the Science Foundation of Beijing Jiaotong University under Grant 2011JBM130. The authors 
would also like to gratefully acknowledge the insightful comments and suggestions by the associate editor and anonymous reviewers.

\section{References}

[1] R. Olfati-Saber and R. M. Murray, "Consensus problems in networks of agents with switching topology and time-delays," IEEE Transactions on Automatic Control, vol. 49, no. 9, pp. 15201533, 2004.

[2] V. Gazi and B. Fidan, "Coordination and control of multi-agent dynamic systems: models and approaches," in Swarm Robotics, E. Sahin, W. Spears, and A. WinfieldParaaaa, Eds., vol. 4433 of Lecture Notes in Computer Science, pp. 71-102, Springer, Berlin, Germany, 2007.

[3] Z. Peng, G. Wen, A. Rahmani, and Y. Yu, "Leader-follower formations control of nonholonomic mobile robots based on a bioinspired neurodynamic based approach," Robotics and Autonomous Systems, vol. 61, no. 9, pp. 988-996, 2013.

[4] Z. Peng, G. Wen, A. Rahmani, and Y. Yu, "Distributed consensus-based formation control for multiple nonholonomic mobile robots with a specified reference trajectory," International Journal of Systems Science, 2013.

[5] G. Wen, Z. Peng, Y. Yu, and A. Rahmani, "Planning and control of three-dimensional multi-agent formations," IMA Journal of Mathematical Control and Information, vol. 30, no. 2, pp. 265284, 2013.

[6] X. Yang and J. Wang, "Distributed robust $H_{\infty}$ consensus control of multiagent systems with communication errors using dynamic output feedback protocol," Mathematical Problems in Engineering, vol. 2013, Article ID 979087, 12 pages, 2013.

[7] H. Xia, T.-Z. Huang, J.-L. Shao, and J.-Y. Yu, "Formation control of second-order multiagent systems with time-varying delays," Mathematical Problems in Engineering, vol. 2014, Article ID 764580, 8 pages, 2014.

[8] C. W. Reynolds, "Flocks, herds and schools: a distributed behavioral model," ACM SIGGRAPH Computer Graphics, vol. 21, no. 4, pp. 25-34, 1987.

[9] T. Vicsek, A. Czirók, E. Ben-Jacob, I. Cohen, and O. Shochet, "Novel type of phase transition in a system of self-driven particles," Physical Review Letters, vol. 75, no. 6, pp. 1226-1229, 1995.

[10] A. Jadbabaie, J. Lin, and A. S. Morse, "Coordination of groups of mobile autonomous agents using nearest neighbor rules," IEEE Transactions on Automatic Control, vol. 48, no. 6, pp. 988-1001, 2003.

[11] W. Ren and R. W. Beard, "Consensus seeking in multiagent systems under dynamically changing interaction topologies," IEEE Transactions on Automatic Control, vol. 50, no. 5, pp. 655661, 2005.

[12] W. Yu, J. Cao, G. Chen, J. Lu, J. Han, and W. Wei, "Local synchronization of a complex network model," IEEE Transactions on Systems, Man, and Cybernetics B: Cybernetics, vol. 39, no. 1, pp. 230-241, 2009.

[13] J. Liang, Z. Wang, Y. Liu, and X. Liu, "Global synchronization control of general delayed discrete-time networks with stochastic coupling and disturbances," IEEE Transactions on Systems, Man, and Cybernetics B: Cybernetics, vol. 38, no. 4, pp. 1073$1083,2008$.

[14] J. Cao, G. Chen, and P. Li, "Global synchronization in an array of delayed neural networks with hybrid coupling," IEEE
Transactions on Systems, Man, and Cybernetics B: Cybernetics, vol. 38, no. 2, pp. 488-498, 2008.

[15] W. Yu, G. Chen, M. Cao, and J. Kurths, "Second-order consensus for multiagent systems with directed topologies and nonlinear dynamics," IEEE Transactions on Systems, Man, and Cybernetics B: Cybernetics, vol. 40, no. 3, pp. 881-891, 2010.

[16] J. Qin, W. X. Zheng, and H. Gao, "Consensus of multiple second-order vehicles with a time-varying reference signal under directed topology," Automatica, vol. 47, no. 9, pp. 19831991, 2011.

[17] S. Khoo, L. Xie, and Z. Man, "Robust finite-time consensus tracking algorithm for multirobot systems," IEEE/ASME Transactions on Mechatronics, vol. 14, no. 2, pp. 219-228, 2009.

[18] L. Gao, X. Zhu, W. Chen, and H. Zhang, "Leader-following consensus of linear multiagent systems with state observer under switching topologies," Mathematical Problems in Engineering, vol. 2013, Article ID 873140, 12 pages, 2013.

[19] Z. Meng, W. Ren, Y. Cao, and Z. You, "Leaderless and leaderfollowing consensus with communication and input delays under a directed network topology," IEEE Transactions on Systems, Man, and Cybernetics B: Cybernetics, vol. 41, no. 1, pp. 75-88, 2011.

[20] Y. Cao and W. Ren, "Distributed coordinated tracking with reduced interaction via a variable structure approach," IEEE Transactions on Automatic Control, vol. 57, no. 1, pp. 33-48, 2012.

[21] K. Peng and Y. Yang, "Leader-following consensus problem with a varying-velocity leader and time-varying delays," Physica A: Statistical Mechanics and Its Applications, vol. 388, no. 2-3, pp. 193-208, 2009.

[22] H. Su, G. Chen, X. Wang, and Z. Lin, "Adaptive second-order consensus of networked mobile agents with nonlinear dynamics," Automatica, vol. 47, no. 2, pp. 368-375, 2011.

[23] H. Shi, L. Wang, and T. Chu, "Virtual leader approach to coordinated control of multiple mobile agents with asymmetric interactions," Physica D: Nonlinear Phenomena, vol. 213, no. 1, pp. 51-65, 2006.

[24] H. Xia, T.-Z. Huang, J.-L. Shao, and J.-Y. Yu, "Second-order leader-following consensus of multiagent systems with time delays," Mathematical Problems in Engineering, vol. 2013, Article ID 505434, 8 pages, 2013.

[25] L. Gao, J. Zhang, and W. Chen, "Second-order consensus for multiagent systems under directed and switching topologies," Mathematical Problems in Engineering, vol. 2012, Article ID 273140, 21 pages, 2012.

[26] Y. Hong, G. Chen, and L. Bushnell, "Distributed observers design for leader-following control of multi-agent networks," Automatica, vol. 44, no. 3, pp. 846-850, 2008.

[27] W. Yu, L. Zhou, X. Yu, J. Lu, and R. Lu, "Consensus in multiagent systems with second-order dynamics and sampled data," IEEE Transactions on Industrial Informatics, vol. 9, no. 4, pp. 2137-2146, 2013.

[28] K. Liu, G. Xie, W. Ren, and L. Wang, "Consensus for multiagent systems with inherent nonlinear dynamics under directed topologies," Systems \& Control Letters, vol. 62, no. 2, pp. 152-162, 2013.

[29] G. Wen, Z. Duan, W. Yu, and G. Chen, "Consensus of secondorder multi-agent systems with delayed nonlinear dynamics and intermittent communications," International Journal of Control, vol. 86, no. 2, pp. 322-331, 2013.

[30] G. Wen, Z. Duan, W. Yu, and G. Chen, "Consensus of multiagent systems with nonlinear dynamics and sampled-data 
information: a delayed-input approach," International Journal of Robust and Nonlinear Control, vol. 23, no. 6, pp. 602-619, 2013.

[31] J.-L. Wang and H.-N. Wu, "Leader-following formation control of multi-agent systems under fixed and switching topologies," International Journal of Control, vol. 85, no. 6, pp. 695-705, 2012.

[32] Q. Song, J. Cao, and W. Yu, "Second-order leader-following consensus of nonlinear multi-agent systems via pinning control," Systems \& Control Letters, vol. 59, no. 9, pp. 553-562, 2010.

[33] G. Wen, Z. Peng, A. Rahmani, and Y. Yu, "Distributed leaderfollowing consensus for second-order multi-agent systems with nonlinear inherent dynamics," International Journal of Systems Science, 2013.

[34] J. Lü and G. Chen, "A time-varying complex dynamical network model and its controlled synchronization criteria," IEEE Transactions on Automatic Control, vol. 50, no. 6, pp. 841-846, 2005.

[35] J.-L. Wang and H.-N. Wu, "Robust stability and robust passivity of parabolic complex networks with parametric uncertainties and time-varying delays," Neurocomputing, vol. 87, pp. 26-32, 2012.

[36] F. R. K. Chung, Spectral Graph Theory, American Mathematical Society, Providence, RI, USA, 1997.

[37] B. E. Paden and S. S. Sastry, "A calculus for computing Filippov's differential inclusion with application to the variable structure control of robot manipulators," IEEE Transactions on Circuits and Systems, vol. 3, no. 1, pp. 73-82, 1987.

[38] D. Shevitz and B. Paden, "Lyapunov stability theory of nonsmooth systems," IEEE Transactions on Automatic Control, vol. 39, no. 9, pp. 1910-1914, 1994.

[39] R. A. Horn and C. R. Johnson, Matrix Analysis, Cambridge University Press, Cambridge, UK, 1985.

[40] Z. Meng, Z. Lin, and W. Ren, "Robust cooperative tracking for multiple non-identical second-order nonlinear systems," Automatica, vol. 49, no. 8, pp. 2363-2372, 2013. 


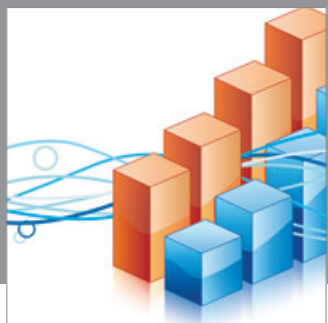

Advances in

Operations Research

mansans

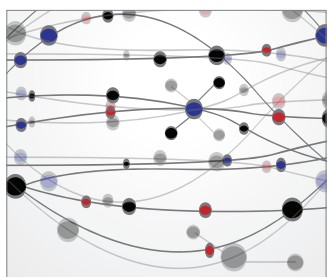

The Scientific World Journal
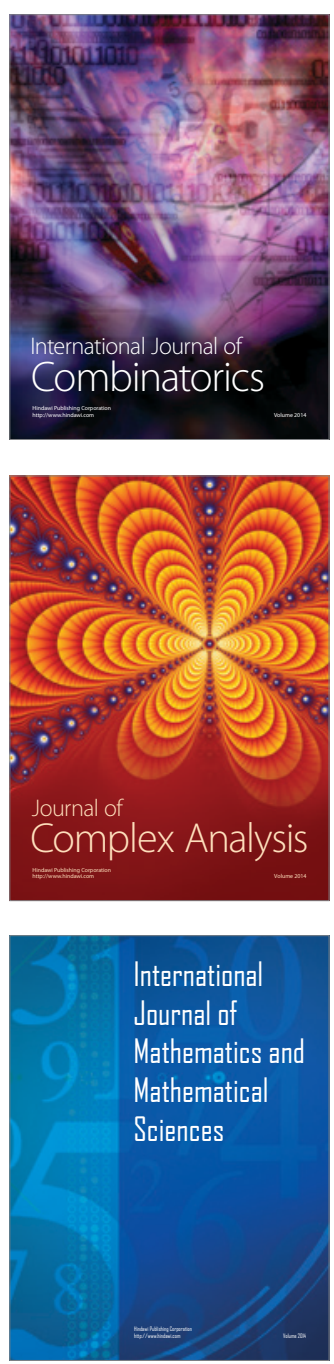
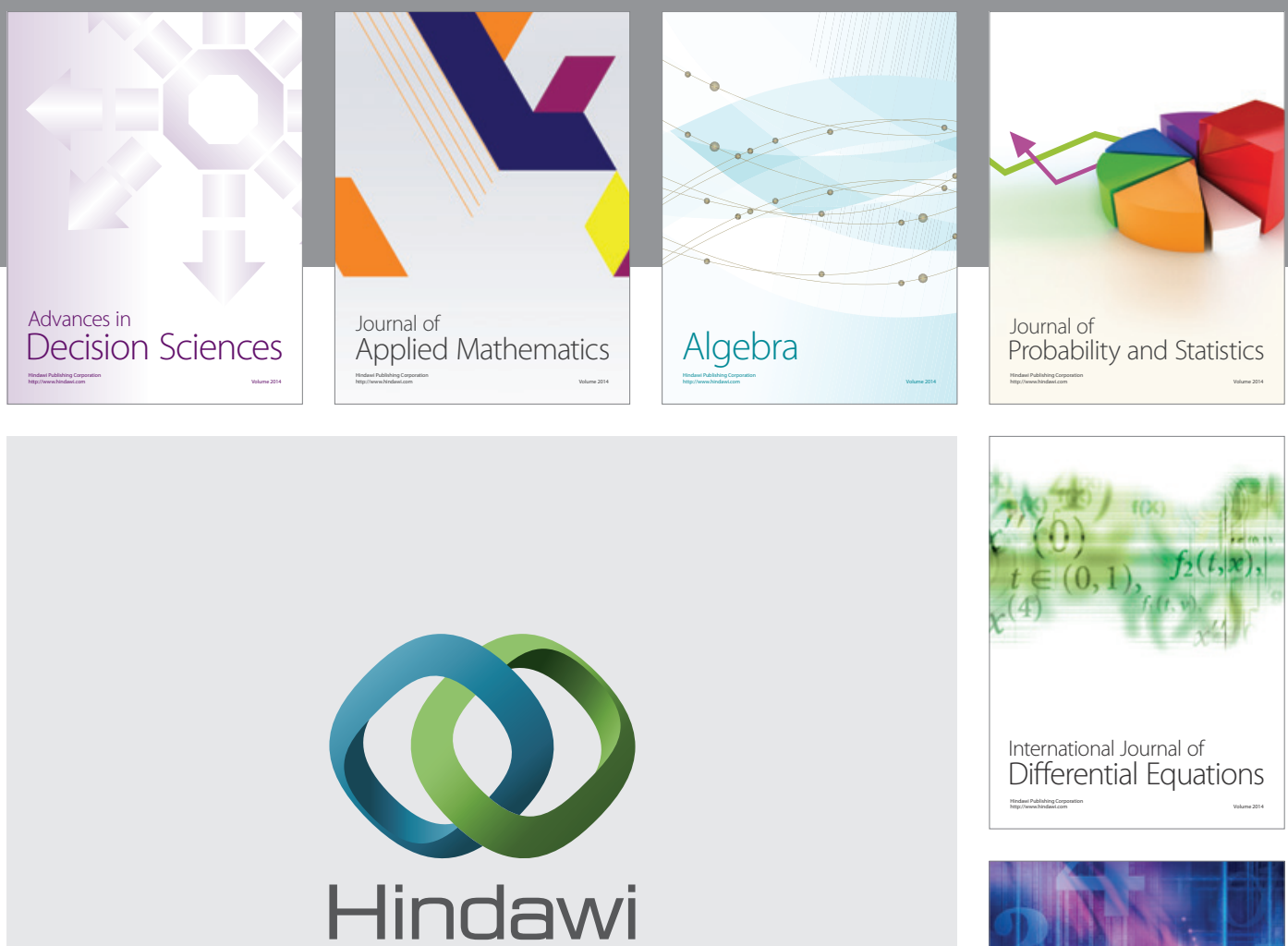

Submit your manuscripts at http://www.hindawi.com
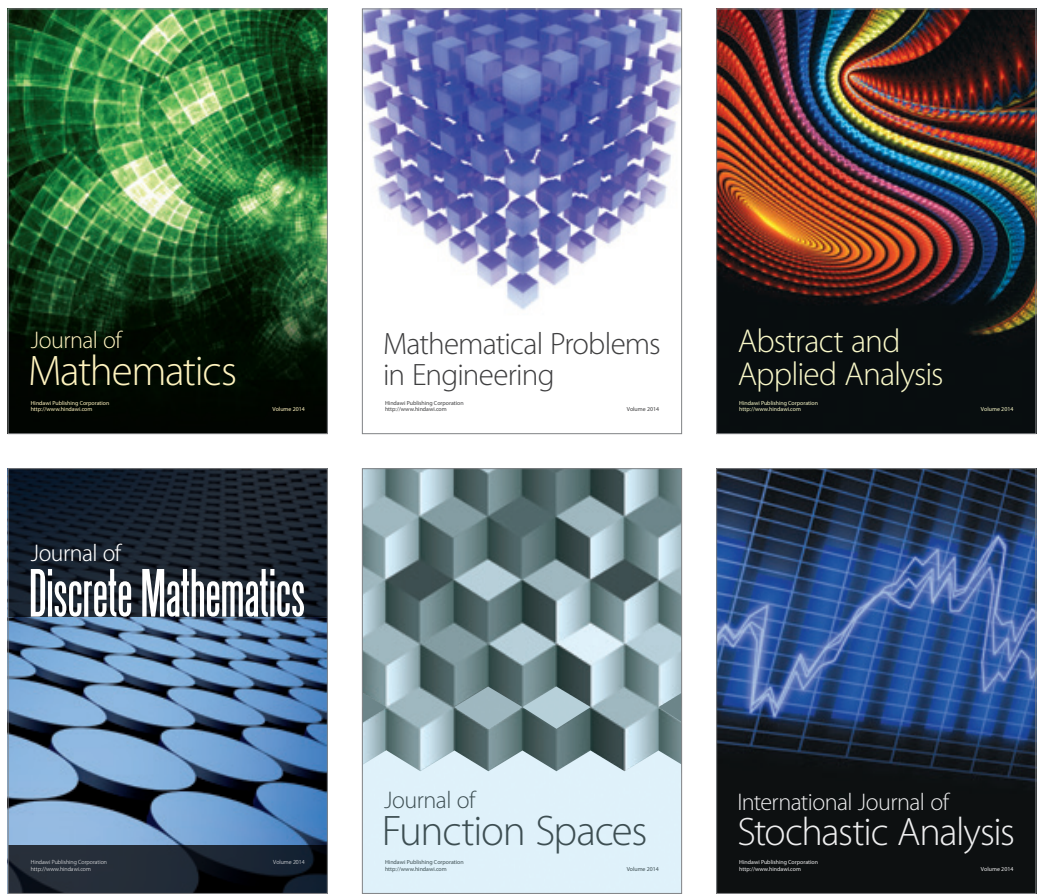

Journal of

Function Spaces

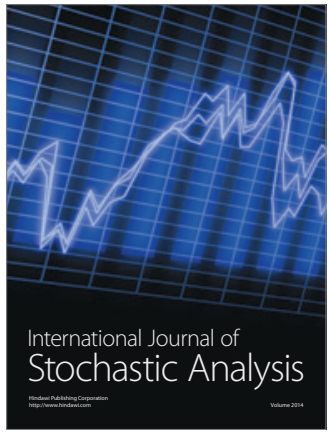

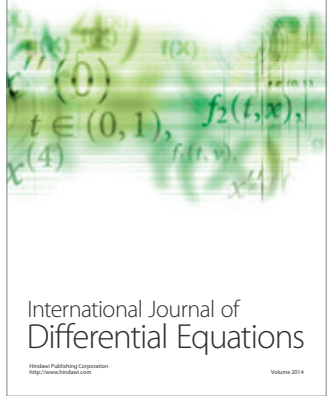
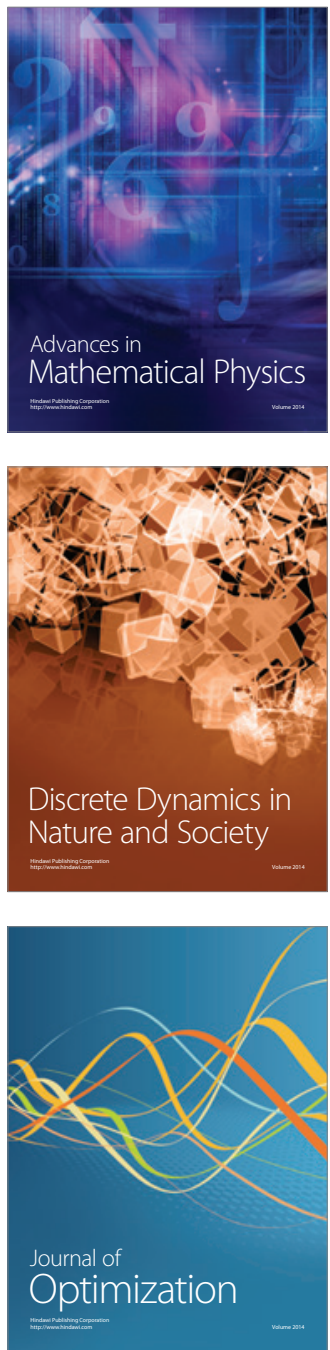